\title{
INFORMATION TECHNOLOGY IN ASSISTED SURGERY
}

\author{
GIANCARLO FERRIGNO *, ANTONIO PEDOTTI *
}

ABSTRACT. - Computer Aided Surgery is opening new scenarios in medicine aimed to improve the patient's care by advancing the utilisation of computer into surgical practice which have already provided practical application particularly in orthopaedics and neurosurgery.

The recent developments in multimodal 3D medical imaging techniques which are providing with great accuracy and the necessary resolution a clear representation on a multiscale basis of the morphological and functional features of the internal organs and tissues constitute the necessary basis. The interdisciplinary collaboration between engineers and physicians provide new concepts and applications in this field derived from digital modelling and simulation methods for the analysis of complex systems, sensors and motion capture technologies, robotics and mechatronics.

Three main phases are described in this paper.

The preoperative phase where the information derived from multimodal imaging of each patient is processed by proper mathematical models and simulation techniques to identify the best way to operate (surgical planning). The intraoperative phase where the surgeon is assisted by a "virtual fusion" of the preoperative images of the internal organs of the patients with the effective position of operative instruments recorded by proper trackers.

Finally the postoperative phase to evaluate the efficacy of the treatments and the patient's follow up.

* Dipartimento di Bioingegneria - Politecnico di Milano. 


\section{INTRODUZIONE}

I nuovi scenari che caratterizzano la chirurgia guidata dall'immagine sono stati resi possibili grazie ai più recenti sviluppi delle tecniche per immagini diagnostiche, della robotica e dell'Information Technology e sono essi stessi trainanti della ricerca in questo settore. I contributi delle tecnologie elettroniche, dell'informazione e meccaniche alla chirurgia non sono ovviamente volte a sostituire l'opera del chirurgo la cui abilità, conoscenza e capacità di decisione e intervento non è in alcun modo sostituibile, così come la necessità di una supervisione umana in un contesto così delicato e rischioso per la vita del paziente. Essi sono piuttosto rivolti a permettere lo sviluppo di nuove tecniche operatorie, a semplificare e rendere più sicure quelle attuali e a velocizzare e rendere più efficiente la macchina sanitaria in questo settore. Le ricadute delle ricerche sulle tecnologie in sanità si rivolgono sia al Sistema Sanitario Nazionale che vede decrescere i costi dei trattamenti chirurgici e la durata delle degenze e aumentare la produttività, sia al paziente che, con le tecniche sempre più diffuse di chirurgia mininvasiva (MIS), vede ridotto l'impatto degli interventi chirurgici, il tempo di degenza e ottiene una migliore qualità di vita dopo l'intervento.

Già oggi molti interventi, soprattutto in neurochirurgia e ortopedia, ma non solo, sono svolti con maggiore sicurezza e precisione grazie alla computer assisted surgery (CAS) integrata nel sistema della computer integrated surgery (CIS) che da poco sta entrando nelle sale operatorie e si va rapidamente imponendo come uno strumento di grande rilievo per la chirurgia del domani. L'impatto di queste tecnologie sulle attività in sala operatoria è paragonabile e supera il contributo dato dall'introduzione del microscopio operatorio, il quale, dal canto suo, entra ormai a far parte dei componenti della CIS. La CIS prevede tre momenti d'intervento della tecnologia integrata in un'unica filiera completamente informatizzata: il momento preoperatorio, il momento operatorio e la valutazione post operatoria. 


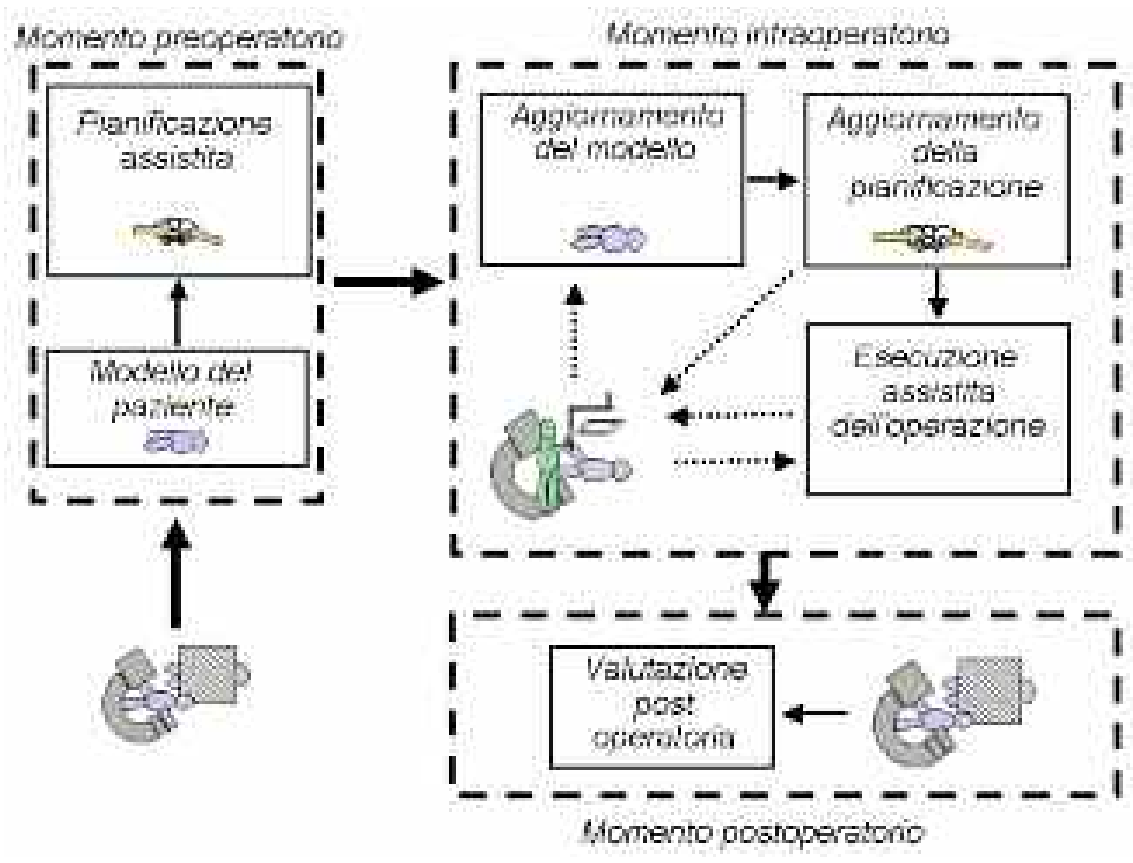

Fig. 1.

I tre momenti principali che contraddistinguono la Computer Integrated Surgery.

\section{PiANIFICAZIONE DELL'INTERVENTO}

Il momento preoperatorio prevede l'acquisizione di informazioni diagnostiche, tipicamente per immagini morfologiche e/o funzionali (CT, MRI, US, SPET, PET, fMRI), che vengono tra loro integrate a formare un modello specifico del paziente. L'integrazione di diverse modalità diagnostiche viene effettuata mediante la cosiddetta messa a registro o registrazione delle immagini. Questa operazione viene effettuata utilizzando informazioni intrinseche o appositamente aggiunte in fase di rilevazione. Nel primo caso vengono identificate delle strutture anatomiche che siano visibili nelle diverse modalità diagnostiche e le immagini vengono rielaborate in modo tale che tali strutture coincidano per forma e posizione su tutte le modalità impiegate. Poiché è però difficile che le stesse strutture siano ugualmente visibili e sufficientemente nitide in immagini rilevate come opacità alle radiazioni ionizzanti o differenza di impedenza agli ul- 
trasuoni, oppure tempi di decadimento di elementi chimici, oppure ancora come attività di sostanze marcanti radioattive, si ricorre a volte all'applicazione di punti di riferimento esterni al corpo (ad esempio fissati al cranio o a caschetti oppure al casco stereotassico) appositamente realizzati per essere osservabili con sufficiente dettaglio in tutte le modalità di imaging previste (Fig. 2). È questo ad esempio il caso di marcatori radiopachi contenenti Gadolinio ben visibile in risonanza magnetica. È chiaro che la maggior semplicità della procedura facente uso di marcatori esterni si scontra con un più pesante carico procedurale e con l'impossibilità di registrare immagini pregresse. Il paziente deve indossare un orpello solidale con la parte del corpo da acquisire. Nel caso più frequente dell'utilizzo nell'imaging del cervello, si utilizza un caschetto, il casco stereotassico o più recentemente dei marcatori radiopachi adesivi. Un altro tipo di elaborazione utilizzato in fase preoperatoria è la ricostruzione di modelli tridimensionali a partire da proiezioni planari multiple. Questa ricostruzione viene ottenuta ponendo in corrispondenza le porzioni di immagine con le stesse intensità di grigio (o di colore in immagini in pseudocolore) e procedendo quindi a una interpolazione. In questo modo la visione della regione operatoria diviene molto più naturale da osservare rispetto alla sequenza di proiezioni. Le problematiche sollevate dalla ricostruzione di modelli tridimensionali risiedono nella possibilità di visualizzazione che richiede tecniche di rendering piuttosto sofisticate. Si procede in genere alla colorazione di alcune strutture evidenziate sulla base della loro differenza di intensità rispetto ai tessuti circostanti. Si tratta in genere di operazioni interattive che mettono in particolare risalto anche l'importanza della user friendliness dell'interfaccia che deve prevedere mezzi di spostamento 3D del modello.
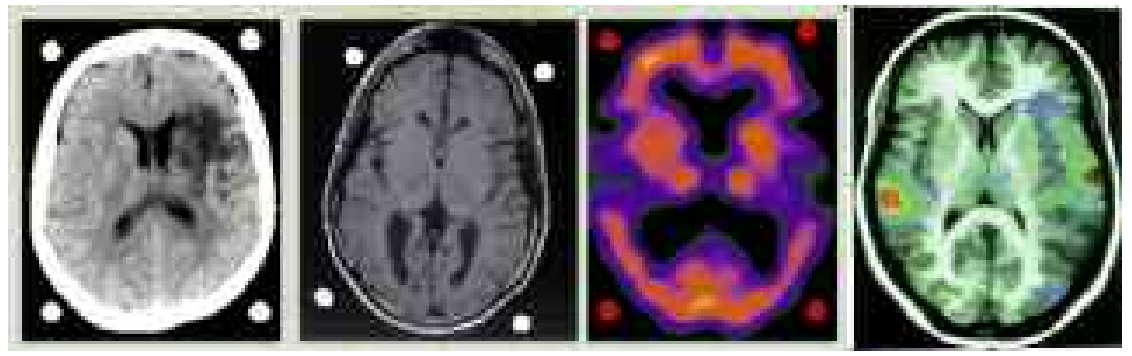

Fig. 2.

Registrazione di immagini ottenute in diversa modalità. 
Sul modello preoperatorio, costituito da informazioni bi e tridimensionali, il chirurgo pianifica la strategia di intervento, pianificazione che può essere effettuata in ambienti di realtà virtuale immersiva per il solo chirurgo, per piccoli gruppi o, utilizzando i più recenti mezzi di proiezione e manipolazione 3D delle immagini, per ampi gruppi di persone. La ricaduta immediata di tale pianificazione si estende facilmente e naturalmente alla fase didattica durante la quale una platea di studenti può assistere ai passi che portano alla pianificazione di una strategia di intervento (Fig. 3). Anche in questi casi l'interfaccia con l'operatore rappresenta un nodo fondamentale di tutto il sistema di realtà virtuale. Si fa ampio utilizzo di mouse multidimensionali equipaggiati con sensori di posizione magnetici a sei gradi di libertà. In questa fase, a differenza del momento della navigazione, l'accuratezza di posizionamento non è infatti cruciale in quanto può essere facilmente compensata, anche in maniera inconscia, dall'operatore, mentre la completa automatizzazione dei sistemi magnetici che non richiedono grosse precauzioni d'utilizzo (se non si è interessati ad accuratezze di misura spinte) rappresenta una semplificazione procedurale impagabile. Linterfaccia deve consentire di penetrare all'interno del modello e di scomporlo nelle sue parti, per mezzo di comandi semplici, resi intuitivi con l'utilizzo di strumenti virtuali quali puntatori, ancoraggi, piani di sezione eccetera, combinati con rappresentazioni in vari colori e, soprattutto in trasparenza.

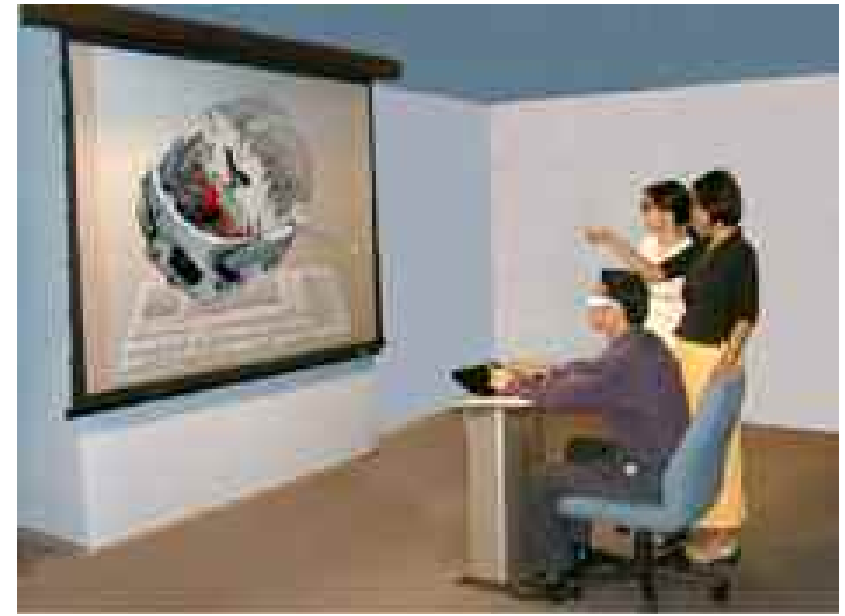

Fig. 3.

Applicazioni didattiche della pianificazione dell'intervento (Dextrobeam di Volume Interactions). 
Una ricaduta secondaria sull'addestramento è la possibilità di utilizzare i modelli preoperatori in sistemi di realtà virtuale con retroazione aptica per simulare interventi chirurgici completamente virtuali (Fig. 4). Le problematiche tipiche di questi sistemi risiedono nella corretta somministrazione all'operatore della retroazione aptica in funzione delle caratteristiche meccaniche del tessuto e degli strumenti utilizzati. È particolarmente facilitato quest'approccio nel caso di interventi in endoscopia ove già l'immagine non è naturale, ma ripresa mediante fibre ottiche e ove il contatto con i tessuti è mediato dalla strumentazione miniaturizzata contenuta nell'endoscopio.

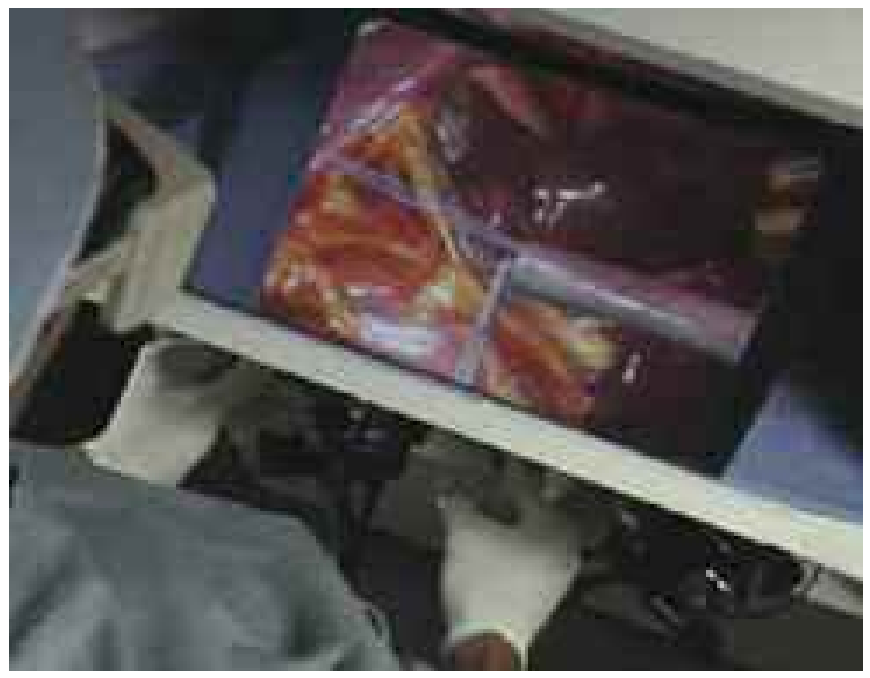

Fig. 4.

Sistema con retroazione aptica per la simulazione di anastomosi di Boston Dynamics.

In questa fase è possibile la trasmissione in rete delle informazioni, non sussistendo vincoli temporali stretti. Tale possibilità viene a cadere quando si lavora in realtà virtuale dove i tempi di presentazione delle informazioni sono particolarmente critici per una corretta percezione. I vincoli che sussistono comunque sulla trasmissione e sull'immagazzinamento delle immagini e di altre informazioni in forma digitale sono relativi a problematiche di sicurezza. Le informazioni non debbono potere essere manomesse senza che di ciò resti evidente traccia, inoltre dati sensibili debbono essere protetti da diffusione fraudolenta. 


\section{ASSISTENZA DAL CALCOLATORE DURANTE L'INTERVENTO}

Nel momento intraoperatorio il chirurgo viene assistito dalle immagini preoperatorie che però possono dare informazioni di gran lunga più utili se possono essere messe in relazione con il corpo del paziente e con gli strumenti chirurgici. La messa a registro delle immagini con il campo operatorio viene effettuata con i cosiddetti tracker, ovvero dei sistemi di rilevazione di marcatori posti sul soggetto e sugli strumenti. La rilevazione dei marcatori consente di posizionare in modo assoluto il paziente nella sala operatoria e di registrare la sua posizione con le immagini preoperatorie. In tal modo un puntatore, anch'esso dotato di marcatori, appoggiato sul capo del paziente, può venire rappresentato nello spazio virtuale delle immagini mostrando la posizione del puntatore in riferimento alle immagini diagnostiche. Così facendo il chirurgo può identificare la posizione di una formazione anatomica fisiologica o patologica dall'esterno del corpo del paziente e operare con una sicurezza maggiore di quella ottenibile dal raffronto delle immagini diagnostiche con la sola visione soggettiva esterna del corpo.

Il tracker è costituito da un sistema stereofotogrammetrico composto da due o più telecamere generalmente operanti nel vicino infrarosso per ridurre il disturbo arrecato allo staff chirurgico. Ogni telecamera è equipaggiata con un flash coassiale con l'obiettivo che illumina i punti salienti contrassegnati con marcatori catarifrangenti. Il catarifrangente si presenta ai sensori delle telecamere come un oggetto estremamente luminoso quando colpito dalla radiazione infrarossa (tra $\mathrm{i}$ 700 e i $900 \mathrm{~nm}$ ) del flash. I marker vengono quindi riconosciuti dalla loro luminosità oppure, in alcuni sistemi, dalla forma circolare. Dei marcatori riconosciuti vengono calcolate le coordinate nel sistema di riferimento sensore (in due dimensioni). La combinazione delle informazioni provenienti da più telecamere permette di calcolare le coordinate dei marker in un sistema di riferimento solidale con la sala operatoria. In Fig. 5 è riportato il modello di un sistema stereofotogrammetrico a due telecamere. La conoscenza di parametri quali le posizioni dei centri di prospettiva ( $C$ e C'), l'orientamento dei due sistemi di riferimento (X, Y, Z e X', Y', Z'), le lunghezze focali $f$ e $f^{\prime}$ degli obiettivi e le coordinate dei punti principali $\mathrm{p}_{0}$ e $\mathrm{p}_{0}$, , consente di ricostruire le coordinate di un punto $\mathrm{P}$ le cui proiezioni misurate siano $\mathrm{p}$ e p' sulle due telecamere. 


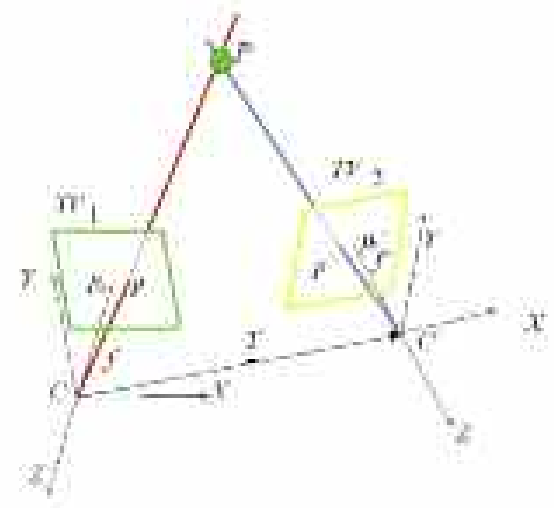

Fig. 5.

Modello teorico di un sistema stereofotogrammetrico.

I punti critici di questo approccio risiedono nella precisione ottenibile con un tracker e la pesantezza di utilizzo in sala operatoria. La Fig. 6 mostra un tipo di sistema a due componenti che cerca di ridurre al massimo l'impatto sulle procedure operatorie richiedendo una visuale libera tra il sistema stereofotogrammetrico, il paziente e gli strumenti chirurgici, consentendo nello stesso tempo di posizionare il monitor che fornisce le informazioni al chirurgo vicino al campo operatorio.

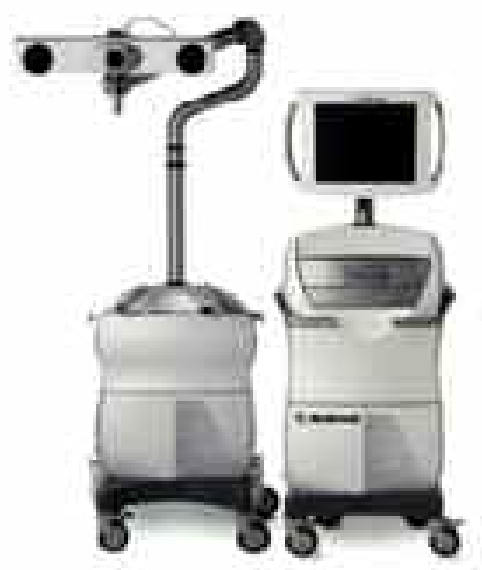

Fig. 6.

Stealth Station della Medtronics. A sinistra il sistema stereofotogrammetrico, a destra il monitor e il sistema di elaborazione. 
Nel momento intraoperatorio sorge un altro problema: le informazioni preoperatorie possono essersi modificate a causa del tempo trascorso oppure, più frequentemente, a causa dell'intervento stesso (ad esempio il brain shift dovuto all'apertura della dura madre con deliquorazione o all'asportazione di parte del tumore). Lo spostamento e la deformazione di organi e tessuti in fase operatoria è tuttora un problema irrisolto che richiede un approccio integrato basato su analisi di immagini intraoperatorie e modelli agli elementi finiti dei tessuti. La soluzione semplice di utilizzare esclusivamente immagini intraoperatorie si scontra con le difficoltà procedurali, il tempo richiesto e il minor dettaglio di alcune delle apparecchiature disponibili per questo scopo. Supponendo comunque di riuscire ad aggiornare il modello del paziente in fase operatoria, il modello della CIS prevede una ripianificazione dell'intervento che richiede soluzioni tecnologiche sufficientemente avanzate da effettuare tale operazione in tempi compatibili con la durata di un intervento chirurgico.

Il terzo contributo della tecnologia nel momento intraoperatorio è la possibilità di eseguire parte dell'intervento utilizzando strumenti robotizzati. Avendo infatti messo in relazione il corpo del paziente con il campo operatorio, è possibile programmare, entro i limiti di precisione del tracker o di altri dispositivi di localizzazione quale il casco stereotassico, movimenti di strumenti montati su bracci robotici. Poiché l'abilità manuale del chirurgo, soprattutto se rapportata con la precisione dei tracker, è più che sufficiente a svolgere correttamente un intervento anche sotto visione al microscopio, la pratica robotica o assistita da robot (per esempio per l'eliminazione del tremore fisiologico, oppure la definizione dei piani di taglio e l'attuazione degli stessi nella inserzione di protesi di ginocchio) è limitata ad alcune applicazioni particolari come la chirurgia funzionale e la chirurgia oftalmica. Recentemente sono stati proposti sistemi integrati comprendenti bracci robotici per il posizionamento di strumenti all'interno del cranio senza fare impiego di casco stereotassico. Sono altresì diffusi sistemi robotici per la realizzazione degli incavi di alloggiamento di protesi d'anca e di ginocchio. La teleoperazione, ovvero l'intervento del chirurgo a distanza, non è proponibile se non per interventi che potrebbero proseguire anche autonomamente, come è il caso di alcune biopsie, e in ogni caso, la trasmissione delle informazioni dovrebbe avvenire su una linea dedicata, non essendo una rete stocastica come internet in grado di garantire tempi di trasmissione determinati, essendo questi dipendenti dal traffico e da altri fattori. 


\section{VALUTAZIONE POSTOPERATORIA E FOLLOW-UP}

Nel momento postoperatorio la CIS continua ad assistere il medico con la valutazione postoperatoria del successo dell'intervento di nuovo sulla base di immagini diagnostiche oppure mediante valutazioni funzionali strettamente correlate con l'intervento effettuato.

Un ultimo contributo che la scienza dell'informazione può fornire alla Medicina è quello della sintesi della storia di ogni singolo paziente su opportuni supporti informatici. La disponibilità degli esami e degli interventi pregressi e di tutta la storia anamnestica del paziente consente di velocizzare le diagnosi future e con esse anche le pianificazioni di eventuali nuovi interventi. Le problematiche tecnologiche di un tale approccio sono essenzialmente legate a problemi di sicurezza e affidabilità del supporto. Servono metodi di codifica dell'informazione sufficientemente sicuri da evitare qualunque possibile manomissione dei dati, anche in vista di un possibile utilizzo in campo legale, e un'affidabilità del supporto, combinata con opportune procedure di backup, che ne garantiscano la leggibilità sul lungo termine.

\section{BIBLIOGRAFIA}

Baroni G., Ferrigno G., Orecchia R., Pedotti A. (2000). Real-time opto-electronic verification of patient's position in breast cancer radiotherapy, Computer Aided Surgery. (vol. 5:4 pp. 296-306).

Baroni G., Ferrigno G., Orecchia R., Pedotti A. (2000). Real-time Three-dimensional motion analysis for patient positioning verification, Radiotherapy And Oncology. (vol. 54 pp. 21-27).

Aliverti A., Dellaca R., Pelosi P., Chiumello D., Pedotti A. (2000). Opto-electronic plethysmography in intensive care patients American Journal Of Respiratory And Critical Care Medicine. (vol. 161 pp. 1546-1552).

Baroni G., Ferrigno G., Pedotti A. (1998). Implementation and application of real-time motion analysis based on passive markers, Medical \& Biological Engineering \& Computing. (vol. 36, n.6 pp. 693-703).

Borghese N.A., Ferrigno G., Ferrari S. (1998). Image Reconstruction using a Hierarchical RBF Network Architecture WIRN, Vietri - 97. pp. 177-182, Perspective in neural computing. ed. by M.Marinaro and R.Tagliaferri, Springer. 
Borghese N.A., Ferrigno G., Baroni G., Ferrari S., Savarè R., Pedotti A. (1998). AUTOSCAN: A flexible and portable scanner of 3D surfaces IEEE Computer Graphics And ApplicationS. (pp. 38-41).

Rizzo G., Gilardi M.C., Prinster A., Grassi F., Scotti G., Cerutti S., Fazio F. (1994). An elastic computerized brain atlas for the analysis of clinical pet/spect data, European Journal of Nuclear Medicine. (vol. 22 pp. 1313-1318).

Cugini U., Bordegoni M., Rizzi C., De Angelis F., Prati M. (1999). Modeling and Haptic Interaction with Non-rigid Materials. Eurographics '99 State of the Art Reports.

Riboldi M., Baroni G., Spadea M.F., Tagaste B., Garibaldi C., Cambaia R., Orecchia R., Pedotti A. (2007). Genetic evolutionary taboo search for optimal marker placement in infrared patient setup. Phys. Med. Biol., 52(19):5815-30. 
